COMMENT. In addition to delays in motor milestones, children with DMD are late in speaking single words and sentences. The late talkers also develop cognitive deficits involving vocabulary. Both late talkers and late walkers function poorly on tests of visuospatial reasoning. Age at taking the first step is generally considered the most reliable milestone based on parents' recollection, while sitting and uttering the first word are less well timed. In the present study, by focusing on broad categories ("on-time" and "late") and by using siblings as controls, the authors have attempted to minimize the limitations of a retrospective study.

Although DMD is primarily a disease of muscle, unexplained language and developmental delay may precede significant signs of muscle weakness, and point to a coincident CNS disorder. Deficiency of brain synaptic dystrophin was demonstrated in an 8year-old child with DMD examined at autopsy (Kim T-W et al. Ann Neurol 1995;38:446449). Several studies have demonstrated the association of cognitive deficits, especially specific verbal impairments in children and adolescents with DMD. The present report shows that delayed language milestones may be the earliest sign of DMD, and an important observation in the clinical diagnosis. The need for further study of brain dysfunction in children with DMD is indicated.

\title{
OUTCOME OF LONG-TERM CORTICOSTEROID TREATMENT IN DUCHENNE MUSCULAR DYSTROPHY
}

The clinical orthopedic effects of chronic daily corticosteroid treatment were evaluated by chart review in boys with genetically confirmed Duchenne muscular dystrophy (DMD) followed at the Ohio State University Muscular Dystrophy Clinic between 2000 and 2003. Becker dystrophy cases diagnosed in 16 were excluded. Of 143 with DMD, 75 (52.4\%) had been treated with steroids for at least 1 year (prednisone $0.75 \mathrm{mg} / \mathrm{kg} /$ day or deflazacort $0.9 \mathrm{mg} / \mathrm{kg} / \mathrm{day})$, and $68(47.6 \%)$ had never been treated or had received brief trials of subtherapeutic doses $(5 \mathrm{mg}$ prednisone daily for $<6$ months). Both cohorts had a mean height below the $5^{\text {th }}$ percentile. Mean age of the treated group was 16.9 years (range 6.1-30.5 years) vs 14.4 (1.1-39.6 years) for untreated patients. Reason for non-treatment was invariably parent fear of side effects. Mean duration of steroid treatment was 8.04 years (range 1-18 years). Independent ambulation was lost at $12.52+/-3.02$ years for steroidtreated vs $9.21+/-1.48$ years in untreated patients $(\mathrm{P}<.0001)$. Lower limb fractures accounted for loss of ambulation in only 1 case $(3.1 \%)$ in the untreated group compared to 11 $(40.7 \%)$ of the steroid-treated patients. Scoliosis of $>10$ degrees developed in $31 \%$ of treated vs $91 \%$ of untreated $(\mathrm{P}<.0001)$. Vertebral compression fractures occurred in $32 \%$ of steroidtreated and none of untreated patients $(\mathrm{P}=.0012)$. The prevalence of femoral fractures was $28.3 \%$ in treated vs $7.27 \%$ in untreated patients (P.0051). Humeral fractures were less frequent in steroid-treated $(9.43 \%)$ compared to untreated patients $(25.45 \%)(\mathrm{P}=.042)$. (King WM, Ruttencutter R, Nagaraja $\mathrm{HN}$ et al. Orthopedic outcomes of long-term daily corticosteroid treatment in Duchenne muscular dystrophy. Neurology May 2007;68:16071613). (Reprints: Dr JT Kissel, Department of Neurology, Division of Neuromuscular Disease, Ohio State University Medical Center, 1654 Upham Dr, Columbus, OH 43210). 
COMMENT. Long-term treatment with steroids in DMD patients results in an increase in duration of independent ambulation of more than 3 years and a significantly decreased risk of scoliosis. Adverse effects include a vertebral compression fracture in onethird of steroid-treated patients, although $80 \%$ are asymptomatic and are discovered incidentally at scoliosis screening. The risk of long bone fracture is 2.6 times greater in treated compared to untreated patients. The increased fracture rate is linked to prolonged independent ambulation and increased body weight. All treated patients are prescribed calcium supplements and have bone density measurements.

\section{DOK7 MUTATIONS IN CONGENITAL MYASTHENIC SYNDROMES}

Clinical and genetic data of 14 patients from 12 congenital myasthenic syndrome (CMS) kinships with mutations in the DOK ('downstream-of-kinase') 7 gene are presented by researchers from the Friedrich-Baur-Institute, Munich, Germany, and several other centers. The chinical presentation of CMS with DOK7 mutations was variable. Typically, onset of symptoms was in the $2^{\text {nd }}$ year of life, with an awkward or waddling gait. Four patients were hypotonic at birth, whereas 3 developed symptoms in the $2^{\text {nd }}$ or $3^{\text {rd }}$ decade. The majority (12 of 14) had bilateral ptosis but no paresis of eye movements. Facial and bulbar weakness occurred in $9(64 \%)$, and several had difficulties in chewing and swallowing. Respiratory function was impaired in $10(71 \%)$, and 2 with onset at birth needed assisted ventilation in the first months of life. Most developed scoliosis, and some had general or selective muscle atrophy. All but 1 had moderate to severe proximal muscle weakness, and a waddling 'sinuous gait' (inward rotation of the knees). Weakness was exercise-dependent, and fluctuated over long periods. In some, symptoms were progressive, with loss of ambulation and respiratory insufficiency in adolescence, whereas in others, severity of symptoms remained constant. AChR antibody testing was negative, and creatine kinase levels were normal. Muscle biopsies showed only nonspecific mild myopathic changes, and no tubular aggregates. Intravenous Edrophonium test was positive in 5 (35.5\%), but none benefited from long-term therapy with esterase inhibitors, and some even worsened. Patients with CMS and mutations in other genes differed from CMS with $D O K 7$ mutations. With CHRNE mutations, eye movements are frequently affected, and with CHAT and RAPSN mutations, episodic apneas occur at birth. In contrast, with $D O K 7$ mutations eye movements are usually spared except for ptosis, and respiratory function shows a progressive deterioration. Limb-girdle myasthenia, unlike DOK7 patients is benefited by esterase inhibitor therapy. (Muller JS, Herczegfalvi A, Vilchez JJ et al. Phenotypical spectrum of DOK7 mutations in congenital myasthenic syndromes. Brain 2007;130:1497-1506). (Respond: Hanns Lochmuller MD, Friedrich-Baur-Institute, Molecular Myology Lab, Marchioninistrasse 17, 81377 Munchen, Germany).

COMMENT. The DOK7 mutation congenital myasthenic syndrome has a broad clinical phenotype, with onset varying from birth to adult life. Features distinguishing the $D O K 7$ mutation patients from other known mutation phenotypes include the absence of external ophthalmoplegia, a progressive deterioration of respiratory function, and lack of long-term response to esterase inhibitors.

Since the description of a congenital myasthenic syndrome in 6 infants treated at the Massachusetts General Hospital in 1960, a variety of molecular causes for the syndrome have 